\title{
On-Chip High-Voltage Sensors Based on Trap-Assisted 2DEG Channel Control
}

\author{
Mohammad Samizadeh Nikoo, IEEE, Member, Armin Jafari, and Elison Matioli, IEEE, Member
}

\begin{abstract}
In this work, we present a new device concept for compact high-voltage sensing with high-impedance input port, consisting of an AIGaN/GaN high-electronmobility channel controlled by trapped carriers generated by a metallic electrode. The high-voltage applied to the metallic electrode is determined by measuring the current of the biased high electron-mobility channel. This approach replaces high-voltage probing with low-current measurement and gives a complete isolation between the high-voltage node and the sensing point. Increasing the electrode-to-channel distance in the device leads to a larger measurable voltage range. The device sensitivity can be increased by decreasing the electrode-to-channel distance or by applying a larger bias to the channel. Devices with extremely large input resistance of $>100 \mathrm{G} \Omega$ in the sensitive region, along with $2 \mathrm{kV}$ breakdown voltage were fabricated in small dimensions of $50 \mu \mathrm{m} \times 50 \mu \mathrm{m}$. The high breakdown field of $\mathrm{GaN}(\sim 3 \mathrm{MV} / \mathrm{cm})$, as a widebandgap material, makes it possible to design and fabricate devices based on this topology for future on- and off-chip high-voltage sensing, providing extremely large input resistances which cannot be achieved by conventional methods.
\end{abstract}

Index Terms-High-voltage sensors, GaN, High electron mobility, input resistance.

\section{INTRODUCTION}

$\mathrm{T}$ HERE is a variety of different applications for high voltage signals, from biology [1] and physics experiments [2] to plasma [3] and vacuum systems [4]. In power transmission and power conversion, using higher voltages immediately results in a higher power delivery. Depending on the application, the required voltage level can vary from a few hundreds of volts to more than $1 \mathrm{MV}$.

Generation and measurement of high-voltages are both challenging. While there are several common techniques to generate high voltages, such as high voltage transformers [5], voltage multiplier circuits [6], and inductive switching [7], high voltage sensing involves additional challenges related to the large structures necessary to control the electric fields, to avoid flashover and also to control the heat dissipation within the circuits [8].

The authors are with the Power and Wide-band-gap Electronics Research Laboratory (POWERlab), École polytechnique fédérale de Lausanne (EPFL), CH-1015 Lausanne, Switzerland (e-mail: mohammad.samizadeh@epfl.ch, elison.matioli@epfl.ch).
There are several high-voltage measurement methods for different voltage levels and termination types. In pulsed-power where high-voltage and high-power come together, it is common to use high-voltage coaxial attenuators, typically 50 $\Omega$-terminated [9]. These attenuators are easy to use and commercially available, up to about $10 \mathrm{kV}$, however, the very high power delivered to the attenuator causes a potential overheating and more importantly, in many cases the highvoltage source is not designed to drive a $50-\Omega$ load.

Voltage dividing is a well-known high-voltage measurement method [10], in which high-voltage is applied to a very large resistor in series with a smaller one, resulting in a large scale factor (e.g. 1000:1). By measuring the voltage over the smaller resistor one can determine the high-voltage based on the nominal scale factor. Despite normally large dimensions of these measurement systems, their input resistance is rather limited, causing self-heating from the dissipated power, which results in a variation in the scale factor [11].

In this letter we propose a new device concept for highvoltage measurement applications, that provides extremely large input resistances, as large as $100 \mathrm{G} \Omega$ or higher. The device takes advantage of the high breakdown field of $\mathrm{GaN}$, to sense high-voltages in small scales, where the device active area can be as small as $50 \mu \mathrm{m} \times 50 \mu \mathrm{m}$. As a wide-bandgap material, $\mathrm{GaN}$ is promising for compact and efficient highvoltage power-electronic systems [13]-[15], and here we show its potential for high-voltage sensing. The device actively measures the applied high-voltage with a low-bias power, in a way that the high-voltage side is isolated from the measurement point and its sensitivity can be increased by applying higher biases. We investigate different parameters of the device geometry, and show that it is possible to design devices with capability of sensing different voltage ranges. These properties together with the simple fabrication of the devices show their great potential for high-voltage probing. The device can also be easily integrated with other components opening opportunities for voltage level monitoring in the future all-on-chip power converters working at high-voltage levels.

\section{Methodology}

Figure 1a shows the cross-section of the proposed device, which consists of an $\mathrm{AlGaN} / \mathrm{GaN}$ high-electron-mobility channel controlled by a metallic electrode, spaced by a distance $\mathrm{g}$ from the channel. The thickness of each layer is 

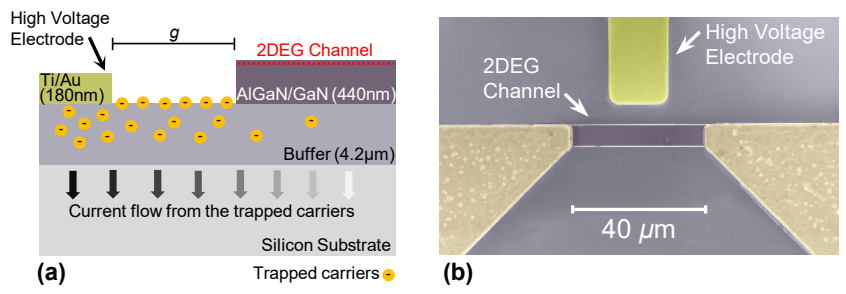

(b)

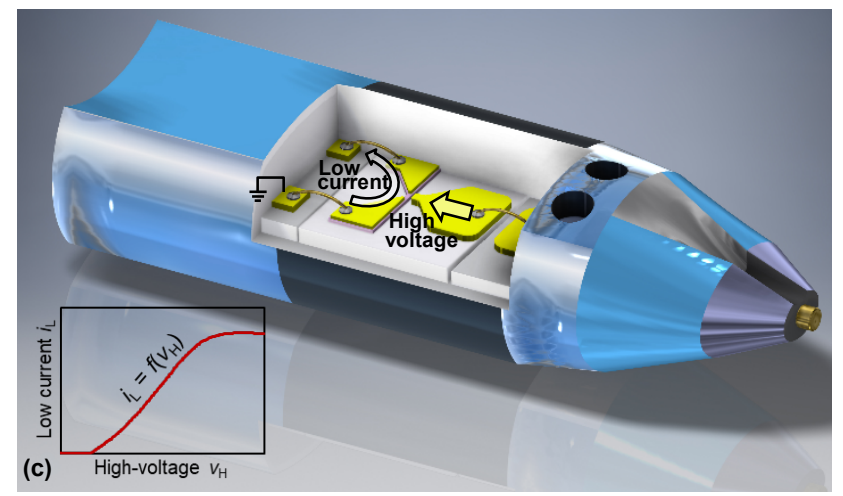

Fig. 1. (a) The cross-section schematic of a high-voltage sensors based on trap-assisted 2DEG channel control along with (b) the SEM image of a fabricated device. (c) A possible way to implement an active probe based on the proposed devices. The high-voltage level is determined by measuring the low-current.

shown in this figure. A top-view SEM image of a fabricated device is shown in Figure 1b. Two terminals of a low-voltage DC source are connected to the high-conductivity 2DEG channel of length $l$ and width $w$ by ohmic contacts [16]. The relatively large distance $g$ together with the large breakdown field of $\mathrm{GaN}$ results in a high breakdown voltage at the input terminal of the devices. The applied voltage to the metallic electrode leads to a small gate current, which charges trap states in the surface and also inside the buffer. These carriers build a potential serving as a back gate to modulate the highelectron-mobility channel. As shown in the inset of Figure 1c, the high-voltage $v_{\mathrm{H}}$ at the electrode controls the conductivity of the channel. Thus the low current $i_{\mathrm{L}}$ is a function $f$ of $v_{\mathrm{H}}$ at a certain channel bias $v_{\text {bias, }}$ which is determined after device calibration:

$$
\dot{\mathrm{L}}_{\mathrm{L}}=\left.f\left(v_{\mathrm{H}}\right)\right|_{v_{\text {bias }}} .
$$

The relation between the high-voltage and low-current makes it possible to determine the applied high-voltage input level from the measurement of the low current $i_{\mathrm{L}}$, by inversing the function $f$. Therefore, this device replaces the measurement of high-voltages by the measurement of low currents. Figure 1c shows a possible way to implement the proposed device as an active probe for high-voltage measurements. The small dimensions of the device together with its relatively large electrode-channel distance lead to a small input capacitance which facilitates charging and discharging of the high-voltage electrode, preventing common surge current issues present in high-voltage sensing methods with large parasitics.

The devices were fabricated on $\mathrm{AlGaN}(19.6 \mathrm{~nm}) / \mathrm{GaN}$ $(420 \mathrm{~nm})$ followed by a $4.2 \mu$ m-thick buffer grown on a 6 -inch silicon substrate. Devices with electrode-channel separation of 2, 6, 10 and $15 \mu \mathrm{m}$, channel length $l$ of $40 \mu \mathrm{m}$ and width $w$ of
2,6 , and $10 \mu \mathrm{m}$ were fabricated to investigate the effect of the device geometry on its operation. The first fabrication step was the definition of the channel by photolithography followed by $\mathrm{Cl}_{2}$-based inductively coupled plasma (ICP) etching to reach the highly resistive buffer layer. The second step was the definition of two ohmic contacts by photolithography. A metal stack of Ti (200 $\AA) / \mathrm{Al}(1200 \AA) / \mathrm{Ti}$ $(400 \AA) / \mathrm{Ni}(600 \AA) / \mathrm{Au}(500 \AA)$ was deposited in the contact regions by electron-beam evaporation and annealed at $780{ }^{\circ} \mathrm{C}$ to form ohmic contacts. The last step of the process was the deposition of $\mathrm{Ti}(300 \AA) / \mathrm{Au}(1500 \AA)$ as the high-voltage input electrode, after photolithography definition.

\section{Results AND Discussion}

Figure 2a shows the functionality of channel current from the electrode voltage for devices with electrode-channel distances of 6,10 and $15 \mu \mathrm{m}$. The measurement was performed for the channel bias $v_{\mathrm{ch}}$ of $2 \mathrm{~V}$. Increasing the electrode-channel distance, reduces the channel control from the electrode, thus requiring higher voltages to modulate the channel electron density. Devices with $g=6$ and $10 \mu \mathrm{m}$ allows the measurement of voltages up to -400 and $-600 \mathrm{~V}$, respectively, while measurement of voltages up to $-850 \mathrm{~V}$ is possible with devices with $g=15 \mu \mathrm{m}$. This gives a general design approach for high-voltage measurements. Figure $2 \mathrm{~b}$ shows these properties in log scale, which reveals that the device acts as a transistor with very large, negative threshold voltage, since the channel can be completely switched OFF with an ON/OFF ratio higher than $10^{8}$. The leakage current from the metallic electrode is shown in Figure 2c, which generally increases at higher voltages, but it is still below $5 \mathrm{nA}$ for input voltages as large as $-850 \mathrm{~V}$. Although all the voltages are negative in these figures, the method is useful for measuring positive voltages by exchanging the ground and signal terminals. In this case, the minus polarity of the channel bias supply $v_{\text {bias }}$ is connected to the high voltage node.
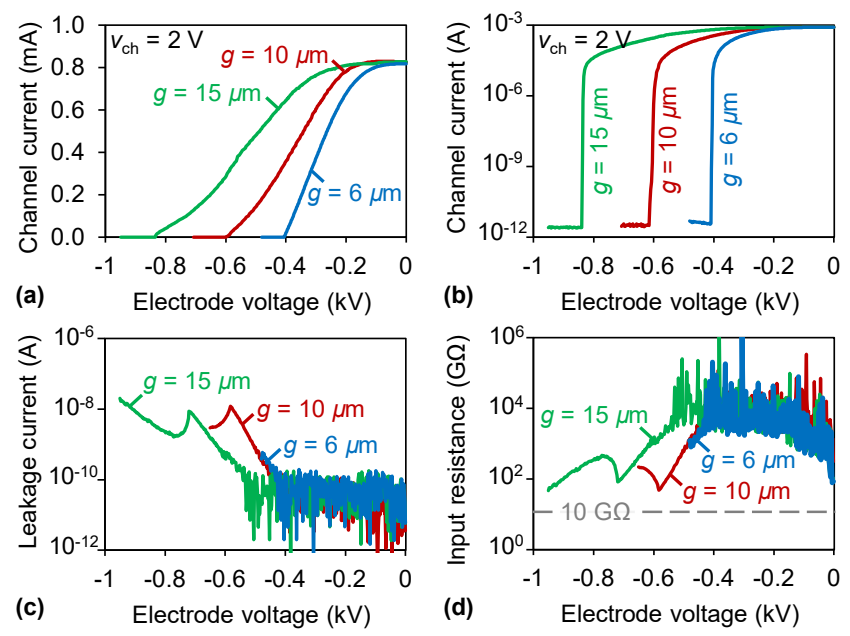

Fig. 2. Functionality of channel current from voltage of the electrode in (a) linear and (b) log scale for electrode-channel distance of 6, 10, and $15 \mu \mathrm{m}$. (c) The electrode leakage current and (d) input resistance of the devices with electrode-channel distance of 6,10 , and $15 \mu \mathrm{m}$. The substrate was floating and the channel bias was $2 \mathrm{~V}$ for all of the figures. 

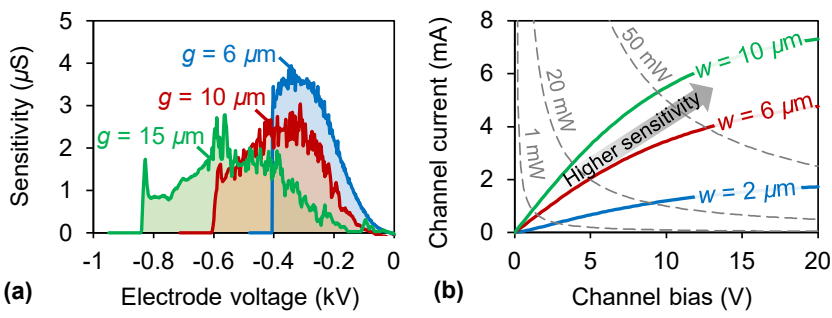

Fig. 3. (a) The sensitivity of the devices with electrode-channel distance of 6,10 , and $15 \mu \mathrm{m}$ versus electrode voltage. (b) The channel current versus voltage bias for three different channel widths of 2,6 , and $10 \mu \mathrm{m}$.

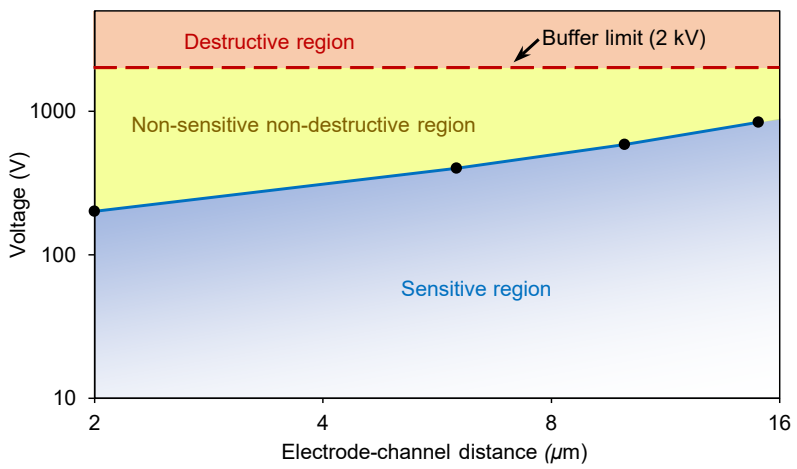

Fig. 4. The device performance in three regions. Below the voltage in which the channel is switched OFF, it is possible to retain the highvoltage level from the channel current. For higher electrode voltages until the breakdown voltage (destructive region), the current is zero and there is no sensitivity.

An important characteristic of a high-voltage measurement device is a large input impedance at high-voltage sensing node, since small impedances may result in significant power dissipation in the measurement tool causing self-heating issues, or disturb the high-voltage source. Figure $2 \mathrm{~d}$ shows the input resistance of devices for different values of the electrode voltage. The input resistances are extremely large, in the range of $10^{13} \Omega$ for a voltage range from $0 \mathrm{~V}$ to $-0.4 \mathrm{kV}$. Increasing the electrode voltage causes a reduction on the input resistance, but it still remains very high. At the limit of the sensitive region, at a voltage level of $-850 \mathrm{~V}$ for the device with $g=15 \mu \mathrm{m}$, the input resistance is greater than $100 \mathrm{G} \Omega$.

Another important parameter of high-voltage sensors, is their sensitivity, which here is defined as the derivative of the channel current with respect to the electrode voltage. Figure 3a shows the sensitivity of the fabricated devices. The area below these curves is equal to the channel current when the electrode is grounded. Therefore, larger values of $g$ result in a larger measurable voltage range and smaller sensitivities. As show in Figure 3a, the sensitivity peaks near the middle of the measurable voltage range and drops for small voltages due to the saturation in channel current. Although low-voltage sensing is not the final goal of these devices, one can improve the device sensitivity by applying a larger bias to the channel, with the downside of a larger power consumption. Wider channel devices yield higher channel currents, however, it does not improve much the sensitivity since the maximum measurable voltage becomes larger. Figure $3 \mathrm{~b}$ illustrates the channel current versus channel voltage for three different channel widths. The contours in this figure show three power consumption levels of 1,20 , and $50 \mathrm{~mW}$. The saturation in channel current occurs due to the saturation in the drift velocity of the carriers in 2DEG.

Based on the electrode voltage, there are three regimes for the proposed sensor (Figure 4). Before depleting the highelectron-mobility channel, there is a one-to-one relationship between high-voltage $v_{\mathrm{H}}$ and low-current $i_{\mathrm{L}}$, defining a sensitive region. In this region, larger values of electrode-tochannel distance are applicable to sense higher voltages. By increasing the electrode voltage, the channel gets depleted and the sensor is saturated (non-sensitive region) until destructive breakdown. In general the breakdown voltage is not a function of the electrode-channel distance, once the channel is off, and it is determined by the buffer thickness. The destructive limit can be improved by either employing a thicker buffer or using GaN-on-GaN epitaxial structures. The robust performance of this sensor together with its flexible and tunable design parameters make the device a promising candidate for highvoltage sensing applications.

\section{CONCLUSION}

We proposed a novel device concept for high-voltage measurement. The device actively probes high-voltage signals by measuring low-currents. The feasibility of the approach was demonstrate by experimental results conducted for devices with different geometries on a GaN-on-Si epitaxy. The fabricated sensors showed a high-voltage performance on small scales thanks to the large breakdown field of GaN. The possibility of designing devices with desired voltage sensitive range and voltage sensitivity was demonstrated. These highvoltage low-power sensors, with large input impedance could be integrated with other power components, opening opportunities for voltage level monitoring in the future all-onchip power converters working at high-voltage levels.

\section{REFERENCES}

[1] H. Hanna, A. Denzi, M. Liberti, F. M. André, and L. M. Mir, "Electropermeabilization of inner and outer cell membranes with microsecond pulsed electric fields: quantitative study with calcium ions," Sci. Rep., vol. 7, p. 13079, Oct. 2017, DOI: 10.1038/s41598-01712960-w.

[2] H. Leea, X. Liu, L. Cultrera, B. Dunhamb, V. O. Kostroun, and I. V. Bazarov, "A cryogenically cooled high voltage DC photoemission electron source," Rev. Sci. Instrum., vol. 89, no. 8, p.083303, Aug. 2018, DOI: $10.1063 / 1.5024954$

[3] R. Snoeckx and B. Annemie, "Plasma technology-a novel solution for $\mathrm{CO}_{2}$ conversion?," Chem. Soc. Rev., vol. 46, no. 19, pp. 5805-5863, 2017. DOI: $10.1039 /$ C6CS00066E.

[4] A. Baig, G. Diana, K. Takuji, A. John, D. Calvi, P. Branko, H. Logan, B. Robert, F. Mar, and C. L. Neville, "Performance of a nano-CNC machined 220-GHz traveling wave tube amplifier," IEEE Trans. Electron Devices, vol. 64, no. 5, pp. 2390-2397, May 2017. DOI: 10.1109/TED.2017.2682159.

[5] R. Prochazka, J. Hlavacek, and K. Draxler, "Magnetic circuit of a highvoltage transformer up to $10 \mathrm{kHz}$," IEEE Trans. Magn. vol. 51, no. 1, Jan. 2015. DOI: 10.1109/TMAG.2014.2357784.

[6] L. Katzir and D. Shmilovitz, "A matrix-like topology for high-voltage generation," IEEE Trans. Plasma Sci., vol. 43, no. 10, Oct. 2015. DOI: 10.1109/TPS.2015.2469101. 
[7] M. S. Nikoo, S. M.-A. Hashemi, and M. Vakilian, "DSRD-based HVdc power supply," IEEE Trans. Plasma Sci., vol. 46, no. 6, Jun. 2018. DOI: 10.1109/TPS.2018.2825438.

[8] E. Kuffel, W. S. Zaengl, and J. Kuffel, High Voltage Engineering Fundamentals, 2nd ed. London, U.K.: Butterworth, 200, p. 77.

[9] C. Li, R. Zhang, C. Yao, Y. Mi, J. Tan, S. Dong, and L. Gong, "Development and simulation of a compact picosecond pulse generator based on avalanche transistorized marx circuit and microstrip transmission theory," IEEE Trans. Plasma Sci., vol. 44, no. 10, Oct. 2016. DOI: $10.1109 /$ TPS.2016.2547944.

[10] R. Marx, "New concept of PTBs standard divider for direct voltages of up to $100 \mathrm{kV}$," IEEE Trans. Instrum. Meas., vol. 50, no. 2, pp. 426-429, Apr. 2001. DOI: $10.1109 / 19.918158$.

[11] Q. Li, L. Wang, S. Zhang, Y. Tang, and Y. Xu, "Method to determine the ratio error of dc high-voltage dividers," IEEE Trans. Instrum. Meas., vol. 61, no. 4, pp. 1072-1078, Apr. 2001. DOI: 10.1109/TIM.2011.2178672.

[12] A. Hariya, T. Koga, K. Matsuura, H. Yanagi, S. Tomioka, Y. Ishizuka, and T. Ninomiya, "Circuit design techniques for reducing the effects of magnetic flux on GaN-HEMTs in $5 \mathrm{MHz}-100 \mathrm{~W}$ high power-density LLC resonant DC-DC converters," IEEE Trans. on Power Electron., vol. 32, no. 8, pp. 5953-5963, Aug. 2017. DOI: 10.1109/TPEL.2016.2616439.

[13] I. C. Kizilyalli, A. P. Edwards, H. Nie, D. Bour, T. Prunty, and D. Disney, "3.7 kV vertical GaN PN diodes," IEEE Electron Device Lett., vol. 35, no. 2, pp. 247-249, Feb. 2014. DOI: 10.1109/LED.2013.2294175.

[14] Y. Zhang, M. Sun, J. Perozek, Z. Liu, A. Zubair, D. Piedra, N. Chowdhury, X. Gao, K. Shepard, and Tomás Palacios, "Large area 1.2 $\mathrm{kV}$ GaN vertical power FinFETs with a record switching figure-ofmerit," IEEE Electron Device Lett., to be published. DOI: 10.1109/LED.2018.2880306.

[15] T. Wang, J. Ma, and E. Matioli, " $1100 \mathrm{~V} \mathrm{AlGaN/GaN} \mathrm{MOSHEMTs}$ with integrated tri-anode freewheeling diodes," IEEE Electron Device Letters, vol. 39, no. 7, pp. 1038-1041, Jul. 2018. DOI: 10.1109/LED.2018.2842031.

[16] D. Delagebeaudeuf and N. T. Linh, "Metal-(n) AlGaAs-GaAs twodimensional electron gas FET," IEEE Trans. Electron Devices, vol. ED29, no. 6, pp. 955, June 1982. DOI: 10.1109/T-ED.1982.20813. 